\title{
Reconversión hospitalaria ante la pandemia de COVID-19
}

\section{Hospital reconversion in response to the COVID-19 pandemic}

\author{
Dra. Cecilia Úrsula Mendoza-Popoca,* Dr. Mario Suárez-Morales*
}

RESUMEN. La reconversión hospitalaria ha sido reconocida como uno de los puntos estratégicos de mayor relevancia a través de la historia de las pandemias. En el 2020, la OMS declaró la presencia de pandemia por COVID-19 el 11 de marzo. En consecuencia en el mundo y en nuestro país, se tomaron las medidas para llevar a cabo la reconversión hospitalaria de acuerdo con las posibilidades y capacidades propias de cada región. La velocidad de reacción requerida para la cristalización de la reconversión agrega un factor más al ya complejo proceso inherente a la misma. Este proceso empieza con el trabajo conjunto de las autoridades hospitalarias y de todo el personal especializado en el diagnóstico, manejo y atención de los pacientes infectados y que pueden llegar a un estado crítico; inicia con el recuento de recursos disponibles, para que a continuación se adapten camas destinadas a otros propósitos para servir como camas de Terapia Intensiva con apoyo ventilatorio, además de asegurarse la participación de personal capacitado e insumos suficientes. En pleno desarrollo de la pandemia hoy en día en nuestro país, la detección de problemas y la toma de decisiones deberán darse conforme a las necesidades cambiantes de cada momento.

ABSTRACT. The hospital transformation is well recognized as one of the paramount strategic points through history in order to prepare for the pandemic appearance. In 2020, the WHO declared COVID-19 a pandemic on March 11. As a result in the world and in our country, measures were taken in accordance to the individual capacity of each region. The speed of reaction for the completion of the transformation adds an extra element that burdens even more, this huge task. This process starts with the conjointly work of the hospital authorities and involves as well all the medical staff and health personnel trained in the diagnostic and treatment of the infected patients, which can reach a critical point in the developing of the disease. The launching of this process begins with the recount of the available resources, continuing with de transformation of hospital beds that were intended for other purposes to function as Intensive Therapy beds along with ventilatory support, besides making sure the participation of prepared health staff and enough medical supplies. In the pandemic development nowadays in our country the detection of the issues and the decision making should be made accordingly to the constant changes of this huge threat.

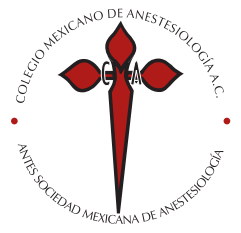

Palabras clave: COVID-19, reconversión hospitalaria, pandemia.

Keywords:

COVID-19, hospital

transformation, pandemic.

* Neuroanestesiólogo. Centro Médico ABC. Ciudad de México.

Solicitud de sobretiros:

Dra. Cecilia Úrsula Mendoza-Popoca Servicio de Anestesiología. Centro

Médico ABC. Campus Observatorio, Sur 136, Núm. 116,

Col. Las Américas, 01120,

Alcaldía Álvaro Obregón,

Ciudad de México.

E-mail: cesa2132@gmail.com

Recibido para publicación: 06-04-2020

Aceptado para publicación: 08-04-2020
L a reconversión hospitalaria es una estrategia de gran relevancia en el proceso de contención y tratamiento de una pandemia, que se desarrolló como consecuencia de la experiencia mundial e histórica, obtenida a partir de la vivencia de previas y catastróficas pandemias.

La magnitud de la incidencia mundial de morbimortalidad que está causando la pandemia de COVID-19 tiene, entre otros antecedentes históricos, la pandemia ocurrida hace un siglo, conocida como la influenza española, la cual puede utilizarse para poner en perspectiva el alcance y las consecuencias de un fenómeno epidemiológico. El estallido de ésta, entre 1918 y 1919, enfermó a uno de cada cuatro estadounidenses y causó la muerte de 675,000 pacientes de una población de $103,208,000$, lo que dio una tasa de mortalidad de 6.5\%. En México, con una población, en ese momento, de 14,556,000, fallecieron 300,000 enfermos, con una tasa de mortalidad de $2.3 \%$. De acuerdo con investigaciones recientes, el estimado de la mortalidad global se situó muy cerca de los 50,000,000 de víctimas ${ }^{(1)}$.

La influenza invadió los Estados Unidos en tres olas letales. Una de las ciudades más afectadas fue Baltimore y octubre de 1918 se caracterizó por ser uno de los peores meses en afectación y muerte, ya que, en ese mes, fallecieron más de 3,000 personas, todo lo cual desembocó en una escasez de mano de obra en todos los rubros, incluyendo transportación y entrega de alimentos; los servicios forenses y depósitos de cadáveres fueron rebasados hasta en 10 veces su capacidad.

La disponibilidad de personal de salud también enfrentó un momento de enorme dificultad, ya que una cantidad importante de médicos y enfermeras servían, en ese momento, 
en la primera guerra mundial, lo que llevó a los hospitales a imponer horarios extraordinariamente extensos al personal de salud disponible. De manera paralela, los hospitales existentes estaban ocupados en su totalidad: pasillos, oficinas, porches e, inclusive, cobertizos aledaños a los hospitales.

De acuerdo con la Dra. Monica Schoch-Spana (2001), de la pandemia de 1918 se pueden desprender varias preguntas:

- ¿Los hospitales estarán en capacidad de hacer frente a la gran cantidad de pacientes que confluyan en un período corto de tiempo?

- ¿Hay suficiente personal calificado para enfrentar una crisis infecciosa agresiva? ¿Cómo se van a proteger?

- ¿Hay suficiente equipo y medicamentos para el tratamiento de estos pacientes?

- En la actualidad, ¿el sector salud de todas partes del mundo está mejor preparado que en 1918 para manejar una emergencia sanitaria de esta magnitud o aún mayor?(2)

Los acontecimientos recientes dan mayor importancia y profundidad a dichas preguntas. En diciembre de 2019, inicia un brote incontrolable de un coronavirus nuevo (SARSCoV-2) en la provincia china de Wuhan, para extenderse, después, a China en su totalidad y, más tarde, a prácticamente todo el mundo. En Wuhan, el número de casos se multiplicó de manera asombrosa, con 2,000 casos nuevos confirmados y $>$ 4,000 sospechosos en una semana; $15 \%$ de ellos desarrolló neumonía severa y alrededor de 6\% necesitó soporte ventilatorio, tanto invasivo como no invasivo. En la actualidad, en esa ciudad hay 1,000 pacientes que necesitan soporte ventilatorio, a los que se suman, en promedio, 120 nuevos pacientes diarios que también requieren de este apoyo. En Wuhan existían, antes del brote epidémico, 600 camas de Terapia Intensiva, por lo que el gobierno hizo una reconversión hospitalaria, transformando tres hospitales generales en hospitales de Terapia Intensiva, con un total de 2,500 camas. Estos hospitales fueron exclusivos y especializados en neumonía secundaria a SARS-CoV-2, y fueron equipados con cánulas nasales de alto flujo y ventiladores tanto invasivos como no invasivos. Sin embargo, se sumó un nuevo problema: la escasez de personal de salud entrenado y capacitado para el tratamiento de estos pacientes con una patología de enorme complejidad, además de existir un desconocimiento total de una ruta terapéutica efectiva. El gobierno chino transfirió 600 médicos intensivistas y 1,500 enfermeras, también intensivistas, del resto de China a Wuhan ${ }^{(3)}$.

Una situación similar empezó a suceder, con velocidad, en todo el mundo, pero en algunas regiones los brotes fueron más agresivos y violentos. Sobresale en este aspecto la región nórdica de Italia, Lombardía. Ahí se encontró que la propagación de la infección viral pasó del descubrimiento del caso número uno el 20 de febrero de 2020, a 36 casos en sólo dos días. En esa región de Italia la capacidad de atención de pacientes en Terapia Intensiva es de 720 camas, lo cual representa 2.9\% del total de camas de 74 hospitales. En 48 horas, se convirtieron 15 hospitales, en los cuales se contabilizaron 130 camas nuevas para Terapia Intensiva. A partir del 7 de marzo, se contó con 482 camas más en 55 hospitales. A pesar de este esfuerzo, de acuerdo con el modelo matemático lineal, para marzo 20 de 2020 habrá 869 pacientes con necesidad de atención intensiva, mientras que el modelo exponencial arrojó un resultado de 14,542 posibles pacientes infectados para esa misma fecha ${ }^{(4)}$.

$\mathrm{Al}$ parecer, la realidad se amolda a las predicciones exponenciales y se podría llegar a contabilizar 30,000 pacientes infectados, de los cuales 4,000 tendrán necesidad de atención en Terapia Intensiva a mediados de abril(5).

En un estudio reciente, que se enfoca en el problema de la escasez de recursos, tanto humanos como de equipo médico, en los Estados Unidos, ante la presencia de la pandemia actual, se presenta las siguientes proyecciones y adecuaciones del sistema de salud: existen 85,000 camas de Terapia Intensiva y se dispone de 62,000 ventiladores completos y otros 98,000 ventiladores con funciones básicas limitadas.

Para el manejo de los ventiladores, además de los médicos y enfermeras especializados, se requiere de terapistas respiratorios, los cuales, en un panorama ideal, deben de ser responsables de vigilar, cada uno, cuatro ventiladores, y se cuenta con 76,000 de ellos. En cuanto al personal de enfermería, se calcula 512,000 enfermeras intensivistas que, idealmente, no deben de tener a cargo a más de dos pacientes; en cuanto al personal médico, se ha establecido que la relación es de un médico por 10 a 15 pacientes.

La proyección de la necesidad de Terapia Intensiva durante la pandemia actual es de 960,000 a 3,840,000 pacientes $^{(6,7)}$. Esto retrata claramente que los recursos de los cuales se puede disponer se ven muy en desventaja ante la presencia de una pandemia de dimensiones colosales, como es la actual de COVID-19.

Por lo tanto, la necesidad de destinar los recursos disponibles, tanto de personal de salud como de implementos médicos de todas las categorías, da lugar a la exigencia de emplear un método para utilizarlos de la forma más eficiente posible, de modo que la mayor cantidad de pacientes pueda ser atendida, principalmente los que se encuentren en estado grave y con la necesidad de ser admitidos en Unidades de Cuidados Intensivos y para los cuales sea imprescindible el uso de un ventilador.

Ante la realidad de la insuficiencia de estos recursos, sobresale la obligación de tener una guía para determinar qué paciente, en el momento de la saturación de servicios médicos, deberá o no recibir la oportunidad de ser admitido en la Unidad de Terapia Intensiva y, a su vez, probablemente ocupará un ventilador. Este problema ya había sido estudiado 
por muchos autores, dada su complejidad, y las guías han sido numerosas. Sin embargo, confluyen, al final, en principios aceptados universalmente y se coincide en que existe, sin duda, la obligación de salvar el mayor número de vidas posibles, así como de aumentar el número de años de vida, tomando muy en consideración el principio de ciclo de vida, según el estadío de la misma, como un criterio fundamental.

Un aspecto sobresaliente es la capacidad de decidir si el paciente en cuestión va a sobrevivir como consecuencia del tratamiento en la terapia intensiva, para lo cual se ha propuesto en forma universal, por su validez comprobada, la escala de SOFA (Sequential Organ Failure Assessment) para adultos; para lo población pediátrica se recomienda el uso de la escala PELOD-2 (Pediatric Logistic Organ Dysfunction 2).

Aunado a lo anterior, debe de tomarse en cuenta la perspectiva de años y calidad de vida, basándose en los antecedentes de comorbilidades severas del paciente:

- Insuficiencia cardíaca clase IV de NYHA.

- Enfermedad pulmonar avanzada con $\mathrm{VEF}_{1}<25 \%$ y una capacidad pulmonar total $<60 \%$ predicha además de una $\mathrm{PaO}_{2}<55 \mathrm{mmHg}$.

- Enfermedad hepática con un score de Child-Pugh $>7$.

- Trauma severo.

- Enfermedad neuromuscular intratable avanzada.

- Metástasis de enfermedad maligna o tumor cerebral primario de alto grado.

En cuanto a la edad, la primera prioridad deben ser los niños y adultos hasta la edad de 49 años; les siguen, en esta línea, los pacientes que no han vivido una vida completa (50 a 69 años); después, los pacientes que han llegado a los límites altos de una vida (70-84 años) y, por último, los que cuentan con 85 años o más.

Las pacientes que están cursando un embarazo merecen un renglón aparte, ya que se trata de, al menos, dos vidas. Se puede partir de la comprobación del estado del o de los productos mediante una evaluación obstétrica de los ruidos cardíacos fetales, los cuales, de ser normales, adquieren una prioridad sobre una paciente no embarazada.

Los criterios de exclusión propuestos son los siguientes:

- Paro cardíaco recurrente, no presenciado o que no responde a desfibrilación o marcapaso.

- Evento neurológico avanzado e irreversible.

Los autores citan la necesidad de, en algunos casos, tomar con cierta flexibilidad esta guía, utilizando criterios que no están tomados en cuenta en estas generalidades, los cuales pueden ser innumerables ${ }^{(8)}$.

La reconversión hospitalaria trae aparejados múltiples problemas, entre los que se encuentra la necesidad de en- contrar un mayor número de personal de la salud suficientemente preparado, que deberá de disponer, en forma rápida y oportuna, de los implementos que tanto los médicos como el resto del personal tienen pleno derecho a recibir. Esto está contenido en los derechos y responsabilidades de los trabajadores de la salud, publicado por la OMS a propósito del brote de COVID-19.

Los derechos de los trabajadores de la salud incluyen los siguientes puntos, como obligación por parte de sus empleadores:

- Asumir la responsabilidad completa de asegurarse de que todos los implementos preventivos necesarios y las medidas protectoras estén al alcance, para proporcionar seguridad ocupacional y minimizar los riesgos.

- Proveer información, instrucción y entrenamiento que lleven a optimizar la seguridad ocupacional, entre lo que se incluye: entrenamiento en prevención de infección y control de la misma; el uso, colocación y retiro de equipo protector personal.

- Proveer al personal de salud de mascarillas, guantes, goggles, batas, jabón de manos, agua y otros implementos de limpieza, en suficiente cantidad, con el fin de que los trabajadores no tengan que invertir en la compra de cualquiera de estos implementos.

- Familiarizar al personal con la información más reciente respecto al comportamiento del COVID-19, además de proveer las herramientas adecuadas para llevar a cabo el triage, pruebas y tratamiento de los pacientes.

- Proveer de medidas de seguridad apropiadas para mantener seguro al personal de salud.

- Proporcionar un ambiente libre de culpa, en el cual los trabajadores puedan reportar incidentes tales como exposición a sangre o fluidos corporales provenientes del sistema respiratorio o casos de violencia, y adoptar las medidas para el seguimiento y ayuda de las víctimas.

- Asesorar a los trabajadores en reportar, tempranamente, la aparición de síntomas para que permanezcan en casa cuando se haya contraído la infección.

- Mantener un horario de trabajo apropiado, con lapsos de descanso.

- Consultar con personal dedicado a la seguridad ocupacional y notificar a los mismos los casos de enfermedad ocupacional.

- Permitir a los trabajadores ejercer el derecho de retirarse ante alguna situación de trabajo en la que consideren que existe un peligro inminente y serio tanto para su vida como para su salud, y proteger a este trabajador de posibles consecuencias por la decisión tomada.

- No obligar a los trabajadores a regresar a trabajar en un sitio donde ha habido peligro inminente para la vida y la salud, hasta que no se hayan tomado las medidas remediales completas y adecuadas. 
- Cumplir el derecho de compensación, rehabilitación y servicios de tratamiento y curación a los trabajadores infectados por COVID-19, a consecuencia de la exposición en el área de trabajo, considerando esto como una enfermedad ocupacional.

- Proveer servicios de salud mental.

- Facilitar la cooperación entre los patrones y los trabajadores, junto con sus representantes ${ }^{(9)}$.

La reconversión hospitalaria se refiere al proceso por medio del cual diferentes tipos de hospitales se preparan para la atención de pacientes durante una crisis de salud, en este caso, durante la pandemia del COVID-19.

En nuestro país, se lanzó un proyecto de reconversión hospitalaria, anunciado el 29 de marzo de 2020, por el Dr. Gustavo Reyes Terán, titular de la Comisión Coordinadora de Institutos Nacionales de Salud y Hospitales de Alta Especialidad, en el que se anunciaron tres fases para los siguientes hospitales, tanto de la Ciudad de México como del Estado de México:

- Instituto Nacional de Enfermedades Respiratorias «Ismael Cosío Villegas».

- Hospital General de México «Dr. Eduardo Liceaga».

- Hospital Regional de Alta Especialidad Ixtapaluca.

- Hospital General «Dr. Manuel Gea González» (considerado hospital híbrido).

- Hospital Infantil de México «Federico Gómez».

- Hospital Juárez de México.

La primera fase incluye:

- Lista de personal y especialidades.

- Disponibilidad de fuentes de oxígeno y aire (número y utilidad).

- Ventiladores mecánicos, utilidad y cantidad.

- Cantidad de monitores e integridad de sus módulos y sensores.

- Eficiencia de compresor de gases en litros/hora.

- Eficiencia de generador eléctrico y plantas auxiliares.

- Número de camas totales de que se dispondrá en contingencia, hasta la tercera fase.

Al iniciar la afluencia de pacientes con COVID-19, deberán suspenderse los servicios de atención ambulatoria y de quirófano para las cirugías electivas; proyectar, cuando se llegue a 50\% de ocupación, las necesidades de capacitación de más personal para transitar a la fase 2 y desocupar completamente las unidades que participarán.

La segunda fase incluye la atención de pacientes críticos por COVID-19 en la Unidad de Terapia Intermedia, en recuperación quirúrgica, en el área de estancia corta, todas las cuales deben contar con tomas de aire y oxígeno.
En la tercera fase se considera el lleno total de la Unidad de Urgencias, pero se respeta la Unidad de Choque para la atención de pacientes críticos. Se instalan adultos en áreas pediátricas. Se consideran las camas que sólo cuentan con toma de oxígeno.

En la fase 1, como parte de la estrategia, se clasifica a los hospitales en COVID y no COVID; entre estos últimos, se considera los siguientes institutos: Instituto Nacional de Cancerología, Instituto Nacional de Cardiología «Ignacio Chávez», Instituto Nacional de Pediatría, Instituto Nacional de Rehabilitación e Instituto Nacional de Perinatología; sin embargo, en etapas más críticas, todos los hospitales podrían atender pacientes diagnosticados con COVID.

Un punto muy relevante es contabilizar el número de camas para cada fase:

- En la fase 1: 117 camas.

- En la fase 2: 421 camas más.

- En la fase 3: 745 camas añadidas.

- Dando como resultado un total de 1,283 camas posibles.

En el resto del país y en forma paralela, otras instituciones, tales como el IMSS, el ISSSTE, Defensa Nacional, la Secretaría de Marina, la Cruz Roja, presentaron también su plan de reconversión, con lo que la Secretaría de Salud manifestó que, en conjunto, en el país, se podría contar, en la fase 2, con 5,059 camas y, en la fase 3, se podría añadir 2,342 camas nuevas de cuidados intensivos; en lo referente a ventiladores, por el momento se cuenta con 3,983.

Respecto al personal de salud para la fase 2 , son necesarios 6,845 médicos. Para la fase 3, hacen falta 6,666 médicos más y también 23,119 enfermeras ${ }^{(10)}$.

En el caso de que la extensión de la pandemia requiera disponer de más unidades médicas para la atención de los pacientes, el IMSS sugiere la siguiente interacción estratégica:

Para atención hospitalaria se recurriría a unidades médicas no IMSS, hospitales temporales de construcción ligera, hospitales arrendados, unidades reconvertidas IMSS, hospitales subrogados y hospitales IMSS COVID-19 nuevos.

Para la atención ambulatoria, se habilitaría unidades móviles IMSS, Centros de Seguridad Social y tiendas IMSS.

Asimismo, existen elementos de interacción sectorial en atención médica con base en acuerdos entre las diferentes instituciones de salud del país (ISSSTE, INSABI, Secretaría de Salud y Pemex), en los cuales se trazan las siguientes metas:

- Regionalización de atención de urgencias.

- Convenios de colaboración.

- Infraestructura compartida ${ }^{(11)}$.

Cuando se ha decidido realizar la reconversión en un hospital, también es indispensable la asignación de funcio- 
nes y responsabilidades para cada servicio y sus respectivos mandos.

La determinación inicial corresponderá al director general de cada hospital, quien iniciará la orden, supervisión y coordinación de las diferentes acciones para dicha reconversión; además de establecer las distintas localizaciones de triage externo para la correcta clasificación y canalización adecuada y segura de los pacientes que lo requieran. También es su responsabilidad asegurarse de que el personal que llevará a cabo el triage cuente con el equipo e insumos necesarios.

Según el servicio se enumeran las tareas correspondientes:

- Jefe de la Unidad de Terapia Intensiva: en él recae la función de la reconversión de las diferentes áreas y principales servicios hospitalarios. Dentro de esto se considera la reconversión de áreas críticas en el Servicio de Urgencias (respetando el área de choque). Determina también la reconversión de área de hospitalización a Unidad de Atención a Paciente Crítico. Reúne a médicos especialistas en atención a paciente crítico, así como a anestesiólogos certificados para la intubación y manejo de la ventilación mecánica. Además, determina la reconversión escalonada o inmediata de las diferentes áreas (urgencias, Unidad de Terapia Intermedia, diferentes unidades con monitoreo, recuperación endoscópica, recuperación quirúrgica y unidad de cirugía ambulatoria), según se requiera.

- Jefe de Cirugía: cancelación de cirugía de alta complejidad para evitar la saturación del Área de Terapia Intensiva.

- Jefe Administrativo: deberá asegurase del acopio y distribución de medicamentos, equipo y demás recursos básicos.

- Jefe de Epidemiología: deberá indicar la toma pertinente de muestras para las pruebas específicas. También tendrá comunicación constante con el CENAVESE (Control Nacional de Vigilancia Epidemiológica y Control de Enfermedades), así como el InDRE (Instituto de Diagnóstico y Referencia Epidemiológicos).

- Jefe de Infectología: se encargará de la asesoría y evaluación de los posibles focos infecciosos; dará los lineamientos al laboratorio de infectología para el adecuado procesamiento de las muestras.

- Jefe de Enfermería: organizará y reasignará funciones al personal, de tal manera que cada enfermera o enfermero pueda atender a dos o tres pacientes.

Todas y cada una de estas tareas tienen la finalidad de conseguir que se establezca los siguientes puntos:

- Triage respiratorio: localización externa y/o interna y áreas alternas para su ubicación con la búsqueda de los siguientes datos de alarma:
- Disnea.

- Oximetría de $<94 \%$ aire ambiente.

- Abundantes secreciones.

- Taquipnea $>20$ respiraciones por minuto.

- Síndrome pleuropulmonar.

- Hipotensión arterial con presión arterial sistólica $<90$ $\mathrm{mmHg}$, además de la necesidad de suspender o reducir tratamiento antihipertensivo, presión arterial media < $60 \mathrm{mmHg}$, disminución de $40 \mathrm{mmHg}$ de presión arterial sistólica habitual.

- Exacerbación de síntomas cardiovasculares o respiratorios de enfermedades crónicas subyacentes.

- Trastorno del estado de conciencia.

- Vómito o diarrea persistente.

- Descontrol glucémico.

Si el paciente presenta datos de alarma, o al menos un dato de la escala qSOFA (quick Sequential Organ Failure Assessment), debe considerarse el traslado a un centro hospitalario de segundo o tercer nivel. Éste debe realizarse en una ambulancia; no se debe enviar a los pacientes a los centros hospitalarios por sus propios medios ${ }^{(12)}$.

- Salas de espera alternas, diferenciando el resultado del triage.

- Áreas de aislamiento en espacios arquitectónicos definidos y señalizados.

- Liberación escalonada de camas de hospitalización a través de gestión de camas.

- Organización de jornadas de trabajo y distribución del personal.

- Definición de procedimientos asistenciales (manejo no crítico y crítico).

- Soporte extraordinario de insumos, equipo y recursos financieros.

A partir del momento de la reconversión se deben acatar las siguientes disposiciones:

\section{Servicios que deben suspenderse}

- Medicina física y rehabilitación.

- TOUR quirúrgico.

- Estrategias educativas de promoción y grupales de prevención a la salud.

- Terapia psicológica grupal.

- Cursos a personal de salud, con excepción de los relacionados con COVID-19.

- Endoscopía electiva.

- Cualquier tipo de estudio programado.

Servicios que pueden posponerse

- Consulta externa de especialidades. 
- Cirugía electiva ambulatoria y no ambulatoria.

- Estudios auxiliares de diagnóstico.

- Atención de pacientes en clínica de heridas y estomas.

\section{Servicios que deben continuar}

- Hemodiálisis.

- Quimioterapia.

- Hematología.

- Banco de sangre.

- Urgencias.

- Hospitalización.

- Unidad de Cuidados Intensivos.

- Cuidados Intensivos Neonatales.
- Cuidados de Quemados.

- Laboratorio de Análisis Clínicos.

- Imagenología.

- Unidad Tocoquirúrgica ${ }^{(12)}$.

El disciplinado y puntual desarrollo de todos y cada uno de los puntos que conforman la reconversión hospitalaria, contribuirán a la exitosa atención de los pacientes infectados de COVID-19 y a la posible disminución de la duración de esta pandemia.

Es importante recordar que deberán continuarse las medidas pertinentes para actuar ante una eventual reactivación.

\section{REFERENCIAS}

1. Johnson NP, Mueller J. Updating the accounts: global mortality of the 19181920 "Spanish" influenza pandemic. Bull Hist Med. 2002;76:105-115.

2. Schoch-Spana M. "Hospital's full-up": the 1918 influenza pandemic. Public Health Rep. 2001;116 Suppl 2:32-33.

3. Xie J, Tong Z, Guan X, Du B, Qiu H, Slutsky AS. Critical care crisis and some recommendations during the COVID-19 epidemic in China. Intensive Care Med. 2020 Mar 2. doi: 10.1007/s00134-020-05979-7.

4. Grasselli G, Pesenti A, Cecconi M. Critical care utilization for the COVID-19 outbreak in Lombardy, Italy: early experience and forecast during an emergency response. JAMA. 2020. doi: 10.1001/ jama.2020.4031. [Epub ahead of print]

5. Remuzzi A, Remuzzi G. COVID-19 and Italy: what next? Lancet. 2020. pii: S0140-6736(20)30627-9. doi: 10.1016/S0140-6736(20)30627-9.

6. Emanuel EJ, Persad G, Upshur R, Thome B, Parker M, Glickman A, et al. Fair allocation of scarce medical resources in the time of Covid-19. N Engl J Med. 2020 Mar 23. doi: 10.1056/NEJMsb2005114.

7. Ward NS, Afessa B, Kleinpell R, Tisherman S, Ries M, Howell M, et al. Intensivist/patient ratios in closed ICUs: a statement from the Society of Critical Care Medicine Taskforce on ICU Staffing. Crit Care Med. 2013;41:638-645.

8. Daugherty Biddison EL, Faden R, Gwon HS, Mareiniss DP, Regenberg $\mathrm{AC}$, Schoch-Spana M, et al. Too many patients... a framework to guide statewide allocation of scarce mechanical ventilation during disasters. Chest. 2019;155:848-854.

9. World Health Organization. Coronavirus disease (CIVID-19) outbreak: rights, roles and responsibilities of health workers, including key considerations for occupational safety and health. Interim Guidance. 19 March 2020.

10. Secretaría de Salud. Comunicado Oficial 30 Marzo 2020.

11. Dirección de Prestaciones Médicas. IMSS. Plan de preparación y respuesta institucional COVID 19. Atención médica. Marzo 2020.

12. Gobierno de México. Secretaría de Salud. Comisión coordinadora de Institutos Nacionales de Salud y Hospitales de Alta Especialidad. Lineamiento para la atención de pacientes por COVID-2019. 14 de febrero 2020. 